\title{
Análisis de la corrupción a nivel internacional y su relación con los costos asociados en la gestión de los negocios
}

pp. 83-92

\author{
HUGO BRISEÑO-RAMÍREZ ${ }^{a}$ \\ JESSICA RUBIANO-MORENO ${ }^{b}$ \\ ALEJANDRO RODRÍGUEZ-MAGAÑA ${ }^{c}$
}

RESUMEN En el siguiente trabajo se busca probar la hipótesis según la cual la corrupción es fruto del alto costo de la legalidad, al obstaculizar la agilidad para hacer negocios. Se realiza un modelo econométrico con una muestra de 43 países, en el que la variable dependiente es el índice de corrupción, y las variables independientes o explicativas son un índice de costo de la legalidad - compuesto a su vez por cinco indicadores-, y una variable asociada al ingreso. Ambas resultan significativas, tal como lo sugiere la teoría. En conclusión, la corrupción es resultado del excesivo costo de la legalidad y del bajo nivel de ingreso.

PALABRAS CLAVE corrupción, costo de la legalidad, mejora regulatoria, marco institucional.

\section{HISTORIA DEL ARTÍCULO}

¿CÓMO CITAR?:

Briseño Ramírez, H., Rubiano Moreno, J. \& Rodríguez Magaña, A. (2017). Análisis de la corrupción a nivel internacional y su relación con los costos asociados en la gestión de los negocios. Perspectiva Empresarial, 4(2), 83-92. http:// dx.doi.org/10.16967/rpe.v4n2a8

RECIBIDO: 1 de agosto de 2017 APROBADO: 29 de agosto de 2017

CORRESPONDENCIA:

Hugo Briseño-Ramírez, Prolongación Calzada Circunvalación Poniente 49, Zapopan, Jalisco, 45010, México.

a Doctor, jefe de academia de finanzas de la Escuela de Ciencias Económicas y Empresariales en la Universidad Panamericana, México. Correo electrónico: hbriseno@up.edu.mx

b Candidata a Doctor, profesora en la Universidad de Ciencias Aplicadas y Ambientales - UDCA, Colombia. Correo electrónico: jesrubiano@udca.edu.co

c Doctor, Secretario académico de la Escuela de Ciencias Económicas y Empresariales en la Universidad Panamericana, México. Correo electrónico: arodrigu@up.edu.co 


\section{¿CÓMO CITO EL ARTÍCULO? HOW TO CITE THIS PAPER?}

\section{CHICAGO:}

Briseño Ramírez, Hugo, Rubiano Moreno, Jessica y Rodríguez Magaña, Alejandro. 2017. "Análisis de la corrupción a nivel internacional y su relación con los costos asociados en la gestión de los negocios". Perspectiva Empresarial 4(2): 83-92. http:// dx.doi.org/10.16967/rpe. v4n2a8

MLA:

Briseño Ramírez, Hugo, Rubiano Moreno, Jessica y Rodríguez Magaña, Alejandro. "Análisis de la corrupción a nivel internacional y su relación con los costos asociados en la gestión de los negocios". Perspectiva Empresarial 4.2 (2017): 83-92. Digital. http:// dx.doi.org/10.16967/rpe. v4n2a8

\section{Analysis of corruption at the international level and its relationship with associated costs in business management}

ABSTRACT This paper attempts to test the hypothesis according to which corruption is the result of the high cost of legality as it hinders the flexibility to do business. An econometric model is made with a sample of 43 countries, in which the dependent variable is the corruption index, and the independent or explanatory variables are the cost of legality index-composed in turn of five indicators-and one variable associated with income. Both turn out to be significant, as the theory suggests. In conclusion, corruption results from the excessive cost of legality and the low level of income.

KEYWORDS corruption, cost of legality, regulatory improvement, institutional framework.

\section{Análise da corrupção a nível internacional e a sua relação com os custos associados na gestão dos negócios}

RESUMO Neste trabalho busca-se provar a hipótese segundo a qual a corrupção é fruto do alto custo da legalidade, ao bloquear a agilidade para fazer negócios. É realizado um modelo econométrico com uma mostra de 43 países, no qual a variável dependente é o índice de corrupção, e as variáveis independentes ou explicativas são um índice de custo da legalidade - composto por sua vez de cinco indicadores -, e uma variável associada ao ingresso. Ambas resultam significativas, exatamente como sugere a teoria. Em conclusão, a corrupção é resultado do excessivo custo da legalidade e do baixo nível de ingresso.

PALAVRAS CHAVE corrupção, custo da legalidade, melhora regulatória, marco institucional. 


\section{Introducción}

El "combate a la corrupción" es una expresión cotidiana en los discursos de políticos y gobernantes, y es que la rendición de cuentas y las métricas de desempeño de las instituciones públicas son claves para la transparencia del quehacer institucional. A la corrupción se le atribuyen, no sin razón, grandes problemas, como, por ejemplo, la falta de confianza en las instituciones, lo que merma la atracción de inversión. Se han propuesto diversos caminos a fin de combatirla, pues tiene varias aristas, caminos que serán claros cuando logren detectarse las variables que inciden en el nivel de corrupción de un país. En el presente documento se busca evidenciar cómo la dificultad para dar cumplimiento a las leyes en materia de negocios fomenta los actos corruptos, y contribuye a esta idea según la cual medir la corrupción permite mejorar a la sociedad por medio del fortalecimiento de sus instituciones y al mejoramiento de la eficiencia del Estado.

Uno de los elementos situacionales que ha propiciado el aumento de la corrupción es la globalización económica, ya que facilita las asimetrías de poder entre naciones y empresas, lo cual se evidencia en la influencia sobre el diseño de políticas públicas que algunos grupos financieros globales ejercen, lo que lleva a un desgaste del Estado a causa de la generación de una cultura de "lobbies económicos" en el diseño de marcos normativos y procedimientos administrativos (Sasia, 2014). No por ello se debe entender que la globalización implica corrupción, sino que favorece una exposición social de indefensión ante mecanismos más sofisticados y elaborados desde el punto de vista conceptual de desarrollo.

Aunado a esto, se observa que los canales de circulación de productos y servicios (importaciones y exportaciones entre países) se han vuelto a tal punto complejos, que terminan por favorecer la falta de control a los flujos monetarios de dichas transacciones, como, por ejemplo, en los casos específicos de las normativas sobre el secreto bancario y los paraísos fiscales, los cuales se presentan día a día sin que se efectúe una postura radical y de cero tolerancia en los organismos internacionales, desde la cual se implementen leyes que erradiquen estas situaciones (Sasia, 2014). Hasta el momento, se han realizado algunos avances, tales como las normativas de Basilea I, Basilea II y Basilea III. Esta última, vigente desde el 2010, surgió como una respuesta a las necesidades de regulación bancaria desde las iniciativas promovidas en el Foro de Estabilidad Financiera y el G20, tras la crisis subprime.

A nivel empresarial no es muy visible el impacto de las prácticas corruptas, sin embargo, es generalizada su utilización en algunos ámbitos y en países en vía de desarrollo. La práctica más común en el sector privado son los sobornos, los cuales normalmente se ejecutan como una estrategia viable con el objetivo de disminuir los tiempos de los trámites administrativos. No obstante, aunque es visto como un acto insignificante, contribuye al desprestigio de la administración pública y de las reglas del Estado de derecho. Además, genera una percepción negativa de la seguridad jurídica de un país, y a la larga termina por impactar en la consecución de recursos para la inversión de las empresas (Martín-Baumeister, 2014). Las compañías y las personas perciben en el soborno una mayor rentabilidad frente al cumplimiento de la ley y el proceso, al valorar el tiempo de espera para un trámite con mayor magnitud a la erogación del soborno. Por su parte, los reguladores encuentran en la lentitud y la burocracia una forma de favorecer un ingreso a funcionarios que, de otra forma, no podría la propia institución pagar (Zakiuddin, 2001).

El problema de la corrupción radica en que ha venido evolucionando con el tiempo, lo que le ha permitido volverse una "corrupción sistemática" con capacidad de deteriorar los mecanismos de control de un país, lo que dificulta su medición y la detección de las variables que la impulsan, y propicia así la ineficiencia de las medidas que se establecen para combatirla. Aunque se observa que los estilos de administración de los Estados han cambiado en procura de lograr una mejor gestión, también se presentan mayores oportunidades para la corrupción, ya que la tendencia es descentralizar los controles dentro de las instituciones, lo que termina por generar mayores riesgos para que se presenten fraudes (Klitgaard, MacLeanAbaroa \& Parris, 2000).

A fin de abordar esta problemática, en la primera parte del artículo se realiza una revisión del concepto teórico de corrupción y su relación con el costo de la legalidad. En la segunda se describen las variables y las observaciones de la base de datos que se utilizó para desarrollar la hipótesis. Luego, se lleva a cabo un análisis estadístico y se presenta una clasificación de los países estudiados según sus niveles de corrupción, y, por último, 
se expone y valida un modelo econométrico, así como la interpretación de sus resultados.

\section{Corrupción y costo de ser legal}

Aunque la corrupción, por lo general, se percibe como algo negativo, existen autores que señalan que puede tener beneficios (Leff, 1964; Huntington, 1968) al darle una connotación de costo beneficio inmediato, en lugar de una visión de largo plazo. Esto se debe a que los actos corruptos los generan los altos costos de la legalidad $^{1}$ (Ghersi, 2006), los mismos que en ocasiones no tienen mucho sentido y no generan un beneficio social inmediato, y sí detienen proyectos empresariales y de construcción e impiden grandes inversiones nacionales y del extranjero. Es por eso necesario generar marcos institucionales que reduzcan los costos de transacción (North, 1993), $\mathrm{y}$ no marcos institucionales que la fomenten y alimenten, al grado de convertirla en un quehacer intrínseco dentro de las sociedades. Aunque la corrupción podría tomar atajos al camino de la ineficiencia en la regulación, esta genera males muy profundos en la sociedad. Como lo señalan Hodgson \& Jiang (2008, p. 76), “la corrupción reduce los niveles de confianza en las transacciones con las empresas y con el Estado", de manera que se benefician solo unos cuantos. Además, "genera externalidades negativas que atraviesan las fronteras sectoriales, debilitan las normas legales y morales, y facilitan otros actos corruptos" (p. 61), en un efecto multiplicador que corrompe hasta convertirse en lo que se ha denominado un "cáncer social" (Morillo, 2009).

Varios autores están de acuerdo en la definición de corrupción como, "actos en los que el poder del cargo público se usa para beneficio personal de una manera que contraviene las reglas del juego" (Jain, 2001, p. 73). Sin embargo, esta definición recibe críticas, pues se menciona que no solo la corrupción se genera desde los cargos públicos, y no solo genera beneficios privados (Hodgson \& Jiang, 2008). En este artículo se abordará la corrupción, entendida desde un enfoque social, como la acción de corromper o corromperse, al transgredir normas legales y principios éticos mediante la acción humana (Del Castillo, 2001). Sin embargo, no es el

1 Concepto acuñado por Ghersi (1998), con base en Coase (1996). objetivo de este trabajo realizar un análisis de la definición de corrupción, sino más bien encontrar cómo las mejoras en la regulación para gestionar los negocios disminuyen los costos para que estos sean legales y, por lo tanto -además del convencimiento de los autores de aquella máxima según la cual "lo que no se mide no se mejora"-, aportar en la detección de variables que permiten medirla.

De acuerdo con Ghersi (2006), la corrupción incrementa con el costo de la legalidad, y disminuye con un aumento en el ingreso. No solo en razón al ingreso mismo, sino con los antecedentes que llevaron a su incremento, como pueden ser una mayor calidad de empleo, mayor nivel educativo o mejores oportunidades laborales. De esta situación se busca proveer evidencia en el presente artículo, al demostrar la siguiente ecuación:

$$
\operatorname{CORR}_{i}=\beta_{0}+\beta_{1} C L_{i}+\beta_{2} Y_{i}+\varepsilon_{i}
$$

Donde CORR es corrupción, $C L$ es costo de la legalidad y $Y$ es ingreso.

Briseño, Mendieta \& Decle (2014) realizaron un modelo con datos de panel para el caso mexicano, en el cual proporcionan evidencia de cómo el incremento en la facilidad para abrir negocios y el aumento en el ingreso tienen un impacto negativo en la corrupción. En este trabajo se busca demostrar estas hipótesis en un plano internacional, al generar y utilizar un indicador de costo de legalidad robusto que involucre diferentes perspectivas.

\section{Base de datos y su análisis}

En este apartado se explica cómo se construyeron las variables y cómo se obtuvieron las observaciones para realizar el modelo econométrico.

\section{Variables}

En la tabla 1 se presenta un resumen en el que se presentan los conceptos teóricos, las variables proxy de estos conceptos a ser utilizadas en el modelo, la fuente de información de la que se obtuvieron, la forma de calcular los valores y la escala. El concepto a estudiar de corrupción se aterrizará con un índice de corrupción basado en la percepción de expertos. El costo de la legalidad se medirá por medio de un índice con escala de 0 a 100, que incluirá a su vez indicadores tales como los días necesarios para abrir una empresa, el tiempo empleado por ejecutivos de alto rango en 
temas burocráticos, las horas empleadas para el cálculo y pago de impuestos, el porcentaje de impuesto sobre el salario y la carga impositiva a las empresas. A fin de medir el ingreso se utilizan las variables ingreso per cápita, años de escolaridad y calidad educativa medida con la prueba PISA.

Sin embargo, al final solo se utiliza la variable calidad educativa por ser la que genera un mejor ajuste en el modelo. Además, esta última trata de dos realidades. Por una parte, es una variable aproximada del ingreso, ya que está altamente correlacionada con este $(0,58)$; y por otra, es un indicador que mide el nivel de conocimientos de la población. Por tanto, se espera que una sociedad con un mayor ingreso (mayor nivel educativo) sea menos propensa a la corrupción, al tener capacidad de cubrir el costo de la legalidad, así como por tener un nivel de conocimientos mayor. Tal como menciona Bautista (2005), la educación es una variable relacionada con el grado de corrupción social, en el sentido en que a mayor nivel educativo, mayor ingreso, y a mayor ingreso, menor corrupción.

\section{Observaciones}

A pesar de que existe información desde el 2001, se utiliza solo la del 2013 referente a 43 países. Esto debido a que, como menciona el documento, existen algunos datos que, aunque son mínimos, se obtuvieron por extrapolación (IMCO, 2015), lo cual puede generar autocorrelación. Además, tal como lo indica Transparencia Internacional (2013), los datos del índice de corrupción no son comparables año con año, ya que no parten de una base estándar y dependen de la información que presenten los expertos de los países, de manera que pueden variar en razón a la dinámica evolución social. Esta subjetividad es una serie limitante para el indicador de corrupción. Sin embargo, es la información de la que se dispone y, por lo tanto, la más completa y confiable.

El hecho de que sean 43 observaciones es también una limitante si los datos no tienen normalidad. No obstante, si se supera esta prueba, así como la de homocedasticidad y correcta especificación, es posible hacer inferencias de una manera más confiable. Es decir, estos datos son los

TABLA 1. Conceptos teóricos y variables proxy para el modelo

\begin{tabular}{|c|c|c|c|c|}
\hline $\begin{array}{l}\text { CONCEPTO } \\
\text { TEÓRICO }\end{array}$ & VARIABLE PROXY & FUENTE & FÓRMULA & ESCALA \\
\hline $\begin{array}{l}\text { Corrupción } \\
\text { (icorr) }\end{array}$ & Índice de corrupción (icorr) & $\begin{array}{l}\text { Transparencia Internacional en } \\
\text { IMCO (2015) }\end{array}$ & $\begin{array}{l}\text { Basado en la percepción } \\
\text { de expertos }\end{array}$ & $\begin{array}{c}\text { o a } 100 \\
\text { (más es peor)* }\end{array}$ \\
\hline \multirow{5}{*}{$\begin{array}{l}\text { Costo de la } \\
\text { legalidad }(\mathrm{cl})^{\star \star}\end{array}$} & $\begin{array}{l}\text { Dificultad para abrir una } \\
\text { empresa (dan) }\end{array}$ & Doing Business en IMCO (2015) & $\begin{array}{l}\text { Número de días } \\
\text { promedio }\end{array}$ & $\begin{array}{l}\text { Mayor que o } \\
\text { (más es peor) }\end{array}$ \\
\hline & $\begin{array}{l}\text { Tiempo de altos ejecutivos } \\
\text { a burocracia (teb) }\end{array}$ & Fraser Intitute en IMCO (2015) & Índice & $\begin{array}{c}\text { De } 1 \text { a } 10 \\
\text { (más es peor) }\end{array}$ \\
\hline & $\begin{array}{l}\text { Tiempo para calcular y } \\
\text { pagar impuestos (tcpi) }\end{array}$ & Banco Mundial en IMCO (2015) & Promedio de horas & $\begin{array}{l}\text { Mayor que o } \\
\text { (más es peor) }\end{array}$ \\
\hline & $\begin{array}{l}\text { Impuesto sobre el ingreso } \\
\text { (isi) }\end{array}$ & $\begin{array}{l}\text { Institute of Management } \\
\text { Development (IMD) en IMCO } \\
\text { (2015) }\end{array}$ & Porcentaje salario & $\begin{array}{l}\text { Mayor que o } \\
\text { (más es peor) }\end{array}$ \\
\hline & Carga impositiva (ci) & $\begin{array}{l}\text { Foro Económico Mundial en } \\
\text { IMCO (2015) }\end{array}$ & Porcentaje utilidades & $\begin{array}{l}\text { Mayor que o } \\
\text { (más es peor) }\end{array}$ \\
\hline \multirow{3}{*}{ Ingreso $(\mathrm{Y})$} & $\begin{array}{l}\text { Ingreso per cápita } \\
\text { (ypc) }\end{array}$ & Banco mundial (2017) & $\begin{array}{l}\text { PIB per cápita en dólares } \\
\text { para el } 2013\end{array}$ & $\begin{array}{l}\text { Mayor que o } \\
\text { (más es mejor) }\end{array}$ \\
\hline & Escolaridad (esc) & Banco mundial en IMCO (2015) & Años promedio & $\begin{array}{l}\text { Mayor que o } \\
\text { (más es mejor) }\end{array}$ \\
\hline & Calidad educativa (caled) & PISA en IMCO (2015) & $\begin{array}{l}\text { Calificación promedio } \\
\text { pruebas PISA en las } \\
\text { materias de matemáticas } \\
\text { y lectura }\end{array}$ & $\begin{array}{l}\text { Mayor que o } \\
\text { (más es mejor) }\end{array}$ \\
\hline
\end{tabular}

*El indicador presentado en la base de datos del IMCO (2015) va de o a 100, siendo 100 menos corrupción. Por lo anterior se reescaló inversamente de o a 100, siendo 100 mayor corrupción.

**Este indicador se construyó a través de promedios en escala de o a 100, siendo 100 mayor costo de la legalidad. 
mejores que se encontraron, dadas las restricciones de información.

\section{Análisis estadístico}

En esta sección se examina el comportamiento de las variables. Se analiza la media y la mediana, así como los valores máximos y mínimos del conjunto de observaciones, lo cual se aprecian en la tabla 2.

A continuación, se describe de manera general el comportamiento estadístico de cada variable, de acuerdo, exclusivamente, con las observaciones de la muestra:

Corrupción (icorr). El promedio del índice de corrupción es de 51,02, y la mediana de 54,55, lo que indica valores muy bajos, los cuales hacen que la media sea menor. Los valores máximos corresponden a Nigeria, Rusia y Guatemala; los mínimos a Dinamarca, Suecia y Finlandia.

Costo de la legalidad (cl). El puntaje medio del costo de la legalidad es de 29,97, y la mediana es de 28,26 . Los países que resultan con máximos valores en este rubro son Brasil, Argentina y Nigeria, mientras que los que obtienen los mínimos son Suiza, Canadá y Sudáfrica.

Dificultad para abrir una empresa (dan). El número de días promedio para abrir una empresa es 15,31 , y la mediana es 11 , por lo cual existen datos extremos que empujan la media a un valor más alto. Los países en los que se necesitan más días son Brasil, Indonesia y China; mientras los que necesitan menos son Australia, Portugal y Corea del Sur.

- Tiempo de altos ejecutivos a burocracia (teb). La media y mediana de este índice es 3,27 y 3,29 , respectivamente. Los valores más altos corresponden a Guatemala, Rusia y Argentina, mientras que los menores a Japón, Suecia y Alemania.

- Tiempo para calcular y pagar impuestos (tcpi). En promedio, el tiempo para calcular y pagar impuestos es de 287,2 horas, mientras que la mediana es de 218. Brasil, Nigeria y Panamá tienen los máximos; mientras que Suiza, Irlanda y Noruega, los mínimos.

- Impuesto sobre el ingreso (isi). La media y la mediana del porcentaje de impuestos son de 18,09 y 17,41 , respectivamente. Los valores máximos son de Hungría, Dinamarca y Austria; y los menores de Sudáfrica, Indonesia y Tailandia.

- Carga impositiva (ci). El porcentaje promedio de impuestos a las empresas es de 46,11, y la mediana de 42,6. Los países con más carga impositiva son Argentina, Colombia y Brasil; y los de menos son Malasia, Irlanda y Canadá.

- Ingreso per cápita (урс). En promedio, el ingreso per cápita es de 29,787 dólares, con una media de 21,619. Los países de la muestra con más ingreso per cápita son Noruega, Suiza y Australia; y los de menos India, Nigeria y Guatemala.

TABLA 2. Estadística descriptiva de variables

\begin{tabular}{|c|c|c|c|c|c|}
\hline & MEDIA & MEDIANA & $\begin{array}{l}\text { DESVIACIÓN } \\
\text { TÍPICA }\end{array}$ & MÍNIMO & MÁXIMO \\
\hline Índice de corrupción (icorr) & 51,02 & 54,55 & 30,58 & 0 & 100 \\
\hline Costo de la legalidad (cl) & 29,97 & 28,26 & 11,43 & 9,91 & 65,44 \\
\hline Dificultad para abrir una empresa (dan)- días & 15,31 & 11 & 12,12 & 2,5 & 83,6 \\
\hline $\begin{array}{l}\text { Tiempo de altos ejecutivos a burocracia } \\
\text { (teb)- índice }\end{array}$ & 3,27 & 3,29 & 01,55 & 1,10 & 6,01 \\
\hline $\begin{array}{l}\text { Tiempo para calcular y pagar impuestos } \\
\text { (tcpi)-horas }\end{array}$ & 287,2 & 218 & 387,2 & 63 & 2600 \\
\hline Impuesto sobre el ingreso (isi)- porcentaje & 18,09 & 17,41 & 8,03 & 1,75 & 31,35 \\
\hline Carga impositiva (ci)-porcentaje & 46,11 & 42,6 & 15,92 & 24,5 & 108,3 \\
\hline Ingreso per cápita (ypc) & 29,787 & 21,619 & 24,206 & 1,452 & 102,900 \\
\hline Escolaridad (esc) -años & 10,49 & 10,57 & 2,30 & 4,80 & 15,25 \\
\hline Calidad educativa (caled) -puntos promedio & 467,2 & 485,9 & 58,33 & 335,8 & 595,1 \\
\hline
\end{tabular}

Fuente: elaboración propia con apoyo del programa GRETL. 
- Escolaridad (esc). Los años promedio de escolaridad son 10,49, y la mediana es 10,59. Los países con más escolaridad son Suiza, Estados Unidos y República Checa; y los de menos Guatemala, India y Turquía.

- Calidad educativa (caled). El promedio de puntaje de la prueba PISA en matemáticas y lectura es de 467,2, y la mediana es 485,9. Los valores máximos pertenecen a los países de China, Corea del Sur y Japón, mientras que los menores a India, Nigeria y Panamá.

En la tabla 3 se observan las correlaciones para cada par de variables. Como se aprecia, el índice de corrupción tiene una correlación positiva importante con el costo de la legalidad, y negativa con el ingreso per cápita, la escolaridad y la calidad educativa. También se observa que el costo de la legalidad está correlacionado negativamente con el ingreso y con las variables asociadas a educación como aproximaciones del ingreso. Por lo anterior, se utilizará calidad educativa (como aproximación al ingreso), que es la variable con menos correlación con costo de la legalidad. Esto con el propósito de reducir el riesgo de multicolinealidad.

\section{Clasificación de países según sus niveles de corrupción}

El criterio para definir los niveles que se ejemplifican visualmente en la tabla 4, serán las desviaciones estándar alrededor de la mediana (no se tendrá en cuenta la media con el fin de evitar sesgo). A partir de una desviación después de la mediana hacia adelante, expresará una alta corrupción; entre la mediana y la primera desviación estándar, será media; entre la mediana y una desviación estándar menos, significará regular; y a partir de una desviación estándar negativa alrededor de la mediana hacia valores más negativos, se referirá a baja.

A continuación, se muestran los niveles de corrupción y se analiza su relación con el costo de la legalidad y sus componentes. Los datos que se presentan son los promedios de cada rango, y se pueden observar en la tabla 5 . Se percibe que mayores niveles de corrupción están asociados a mayores niveles de costo de la legalidad; a mayor dificultad para abrir una empresa; a más tiempo de ejecutivos de alto rango en temas burocráticos; a más tiempo para calcular y pagar impuestos; y a una mayor carga impositiva. Es curioso notar que no ocurre lo mismo con el nivel de impuesto

TABLA 3. Matriz de correlación

\begin{tabular}{lccccc}
\hline & icorr & cl & ypc & esc & caled \\
\hline Índice de corrupción (icorr) & 1 & 0,62 & $-0,86$ & $-0,66$ & $-0,64$ \\
\hline Costo de la legalidad (cl) & & 1 & $-0,55$ & $-0,60$ & $-0,46$ \\
\hline Ingreso per cápita (ypc) & & 1 & 0,67 & 0,58 \\
\hline Escolaridad (esc) & & & & 1 & 0,59 \\
\hline Calidad educativa (caled) & & & & 1 \\
\hline
\end{tabular}

Fuente: elaboración propia con apoyo del programa GRETL.

TABLA 4. Criterios para definir niveles de corrupción

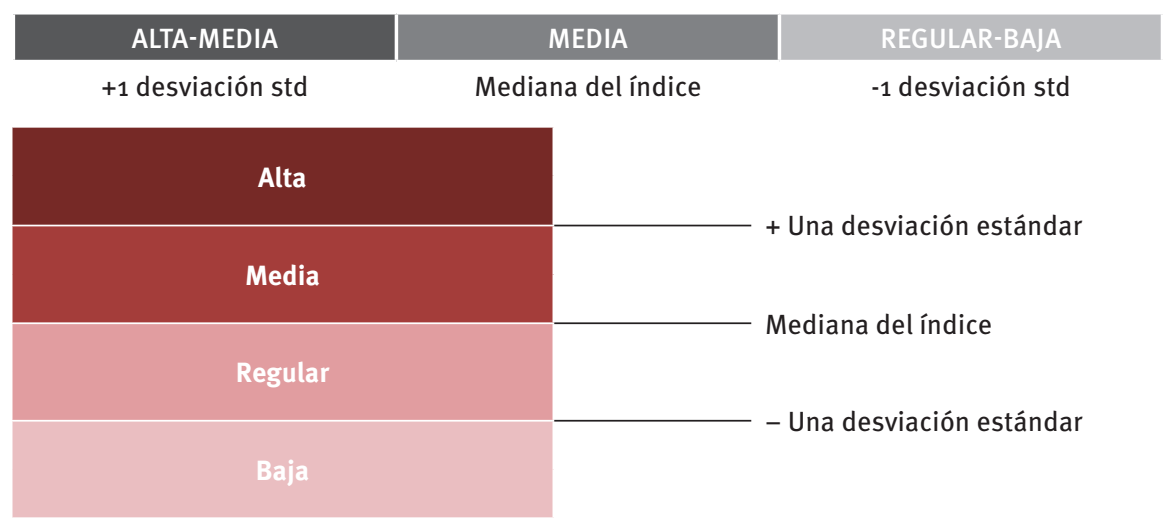


TABLA 5. Indicadores de costo de la legalidad por nivel de corrupción

\begin{tabular}{lrrrr}
\hline NIVEL DE CORRUPCIÓN (icorr) & BAJA & REGULAR & MEDIA & ALTA \\
\hline Costo de la legalidad (cl) & 21,25 & 25,55 & 34,22 & 41,29 \\
\hline Dificultad para abrir una empresa (dan) -días & 8,25 & 10,61 & 19,68 & 24,05 \\
\hline Tiempo de altos ejecutivos a burocracia (teb)-índice & 1,51 & 2,52 & 4,08 & 5,56 \\
\hline Tiempo para calcular y pagar impuestos (tcpi)-horas & 117,8 & 209,27 & 403,87 & 401,48 \\
\hline Impuesto sobre el ingreso (isi)- porcentaje & 22,92 & 19,51 & 15,50 & 14,33 \\
\hline Carga impositiva (ci)-porcentaje & 38,9 & 43,93 & 49,09 & 54,01 \\
\hline
\end{tabular}

Fuente: elaboración propia.

sobre el ingreso; el comportamiento de esta variable es inverso al esperado. Esto se puede explicar al observar cómo, a medida que aumenta la carga impositiva a las empresas, disminuye la carga impositiva a los salarios y viceversa. Además, en algunos países, a mayor ingreso por salario, mayor carga impositiva mediante esquemas de tabuladores de impuestos, lo que se puede interpretar como a mayor carga impositiva, mayor ingreso; a mayor ingreso, mayor nivel de educación; y a mayor educación y mayor ingreso, menor corrupción.

\section{Modelo econométrico e interpretación de resultados}

En este apartado se muestra el modelo con el mejor ajuste. Se revisa su validez de acuerdo con: el cumplimiento de significancia individual, significancia conjunta, normalidad, homocedasticidad, correcta especificación y no multicolinealidad.

TABLA 6. Modelo econométrico (variable dependiente icorr)

\begin{tabular}{lccc}
\hline & COEFICIENTE & ESTADÍsTICO $\mathrm{t}$ & VALOR $\mathrm{p}$ \\
\hline Const & 642,761 & 3,409 & 0,0015 \\
\hline l_cl & 29,7401 & 3,200 & 0,0027 \\
\hline l_caled & $-112,519$ & $-4,012$ & 0,0003 \\
\hline
\end{tabular}

Fuente: elaboración propia con apoyo del programa GRETL.
Se llevaron a cabo varios modelos a fin de explicar la variable índice de corrupción. Se utilizaron como variables explicativas cada uno de los componentes del costo de la legalidad, pero no se tenía un ajuste satisfactorio. Asimismo, se llevaron a cabo regresiones utilizando como variable explicativa el ingreso per cápita o la escolaridad. Sin embargo, los resultados tampoco satisfacían las pruebas estadísticas. El modelo con el mejor ajuste es el que se aprecia en la tabla 6. Se utilizan como regresoras el logaritmo del costo de la legalidad (1_cl), y el logaritmo de la calidad educativa (1_caled); estas regresoras son significativas al uno por ciento. El coeficiente de determinación es de 0,5493 , lo cual indica que prácticamente el $55 \%$ de los cambios en la corrupción se explican por los cambios en las variables independientes del modelo.

En la tabla 7 se observan las pruebas para validar los supuestos. No se rechazan las hipótesis nulas de las pruebas de normalidad, de Reset de Ramsey y de White; por lo que se supone normalidad en los errores, correcta especificación (no faltan variables y la forma funcional es correcta), y homocedasticidad (varianza constante de los errores). Asimismo, la correlación entre las variables explicativas es menor a 0,5, lo que apoya al supuesto de no multicolinealidad.

La tabla 8 muestra las formas funcionales, los coeficientes y las elasticidades, las mismas que se

TABLA 7. Pruebas estadísticas del modelo

\begin{tabular}{llll}
\hline PRUEBA & HIPÓTESIS NULA $\left(\mathrm{H}_{0}\right)$ & VALOR p & INTERPRETACIÓN \\
\hline $\begin{array}{l}\text { Normalidad } \\
\text { (Doornik-Hansen) }\end{array}$ & $\begin{array}{l}\text { El error se distribuye } \\
\text { normalmente }\end{array}$ & 0,2024 & $\begin{array}{l}\text { No se rechaza } \mathrm{H}_{\mathrm{o}} \text {, por lo que suponemos normalidad } \\
\text { en los errores }\end{array}$ \\
\hline $\begin{array}{l}\text { Correcta especificación } \\
\text { Reset de Ramsey) }\end{array}$ & $\begin{array}{l}\text { La especificación es } \\
\text { correcta }\end{array}$ & 0,2284 & $\begin{array}{l}\text { No se rechaza } \mathrm{H}_{\mathrm{o}} \text {, suponemos correcta } \\
\text { especificación }\end{array}$ \\
\hline $\begin{array}{l}\text { Homocedasticidad } \\
\text { (White) }\end{array}$ & $\begin{array}{l}\text { Varianza constante de los } \\
\text { errores }\end{array}$ & 0,2700 & $\begin{array}{l}\text { No se rechaza } \mathrm{H}_{\mathrm{o}} \text {, suponemos varianza constante de } \\
\text { los errores }\end{array}$ \\
\hline
\end{tabular}


TABLA 8. Interpretación de los resultados (variables explicativas de icorr)

\begin{tabular}{lllll}
\hline VARIABLES $X$ 's & $\begin{array}{l}\text { FORMA } \\
\text { FUNCIONAL }\end{array}$ & COEFICIENTES $\beta$ 's & ELASTICIDAD* & INTERPRETACIÓN \\
\hline I_cl & nivel $-\log$ & 29,7401 & $\beta_{2}(1 / Y)=0,5828$ & $\begin{array}{l}\text { Un incremento de } 1 \% \text { en el costo de la } \\
\text { legalidad, disminuye } 0,58 \% \text { la corrupción }\end{array}$ \\
\hline I_caled & nivel $-\log$ & $-112,519$ & $\beta_{2}(1 / Y)=-2,2053$ & $\begin{array}{l}\text { Un aumento de } 1 \% \text { en calidad de la educación, } \\
\text { disminuye } 2,20 \% \text { la corrupción }\end{array}$ \\
\hline
\end{tabular}

Fuente: elaboración propia.

* Se utilizaron las fórmulas que proponen Gujarati \& Porter (2010, p. 173) para obtener la elasticidad en modelos lin-log.

calculan con la fórmula propuesta por Gujarati \& Porter (2010). En este sentido, se muestra evidencia de cómo la corrupción es inelástica ante el costo de la legalidad, lo que significa que los cambios en la primera - debido a los cambios en la segunda- existirán, pero en una proporción menor a los cambios en el costo de la legalidad. Por otra parte, la corrupción es elástica ante la calidad de la educación, lo que muestra que un cambio en la calidad de la educación tiene un impacto más que proporcional en la disminución de la corrupción.

En otras palabras, un incremento de un punto porcentual en el costo de la legalidad disminuye en 0,58 \% el índice de corrupción. Y un aumento de $1 \%$ en la calidad de la educación, disminuye 2,20 \% la corrupción. Así, países que han apostado por un incremento en las inversiones relacionadas con la educación y en programas de mejora en calidad educativa podrían estar construyendo las bases del desarrollo.

\section{Conclusiones}

En este trabajo se explica cómo impactan el costo de la legalidad y el nivel de ingresos en la corrupción. A fin de mostrar evidencia, se construye una variable denominada "costo de la legalidad", con los siguientes indicadores: dificultad para abrir una empresa; tiempo de altos ejecutivos a temas burocráticos; tiempo para calcular y pagar impuestos; y carga impositiva a salarios y utilidades. Se genera una regresión en la cual la variable explicada es el índice de corrupción, y las explicativas son el anteriormente mencionado costo de la legalidad y el nivel de ingresos (expresado en ingreso per cápita, escolaridad o calidad educativa). El modelo que resulta con mejor ajuste utiliza la variable "calidad educativa" como aproximación al ingreso.

Se concluye que el costo de la legalidad tiene un impacto en el aumento de la corrupción, pero de manera inelástica. Es decir, un incremento en el costo de la legalidad tiene un aumento, pero proporcionalmente menor, en la corrupción. En cambio, la corrupción es elástica en cuanto a la calidad educativa. Dicho de otra manera, un aumento en la calidad educativa disminuye la corrupción más que proporcionalmente.

Al considerar la evidencia, se puede señalar que la corrupción se puede disminuir, de manera importante, mediante la reducción en trámites innecesarios, la agilización de los necesarios y la disminución de los costos monetarios de estos. En otras palabras, al disminuir el costo de la legalidad. En cuanto al nivel de ingresos, no se puede concluir demasiado, pues es una variable que no puede modificarse tan fácilmente. Lo que sí se puede decir al respecto es que el nivel educativo provee de herramientas cognitivas y aptitudes que permiten sortear las dificultades que se presentan con los trámites para llevar a cabo negocios. Es decir, es complicado incrementar el nivel de ingresos monetarios, pero sí se puede reducir el costo de la legalidad y proveer herramientas a la población a fin de que supere dichas trabas.

La corrupción deja de ser un tema que se maneja desde la política interna de los Estados, para ser una prioridad en los organismos internacionales. Lo anterior se evidencia en el G-8 y las Naciones Unidas, organismos en los que se realizan convenciones contra la corrupción. No es un tema exclusivo de las naciones no desarrolladas, sino un problema que se extiende a todas las latitudes. Además, los numerosos escándalos que se han presentado a nivel global y afectan a diferentes países, por ejemplo, el caso Odebrecht en América Latina, las pruebas de emisión de contaminantes de Volkswagen en Alemania, o bien el caso de Enron en los Estados Unidos, fomentan las iniciativas académicas para que se estudie el tema y se planteen soluciones que permitan mitigar el impacto de estas prácticas. Es por esto que este tipo de investigaciones intentan abarcar una primera aproximación a un tema que se debe analizar con mayor profundidad. 


\section{REFERENCIAS}

Banco Mundial. (2017). PIB per cápita. Recuperado de: https://datos.bancomundial.org/indicator/ NY.GDP.PCAP.PP.CD

Bautista, O. (2005). La ética y la corrupción en la política y la administración pública (Tesis de maestría). Maestría en Ciencia Política en Iberoamérica, Universidad Internacional de Andalucía.

Briseño, H., Mendieta, R., \& Decle, J. (2014). Corrupción y facilidad para hacer negocios. Un estudio econométrico de panel a nivel municipal para México. En Revista Economía \& Política, 19, 113-142.

Coase, R. (1996). La naturaleza de la empresa (traducido del inglés, 1937). En O. E. Williamson, \& S. G. Winter, La naturaleza de la empresa. Orígenes, evolución y desarrollo (pp. 29-103). México: FCE

Del Castillo, A. (2001, segundo semestre). Fundamentos de la investigación empírica reciente sobre corrupción. Gestión y Política Pública. X(2), 375-402.

Ghersi, E. (1998). El costo de la legalidad. En Estudios Públicos, 30, 83-110.

Ghersi, E. (2006). Economía de la corrupción. Cuadernos Cedice, 73, 3-20.

Gujarati, D., \& Porter, D. (2010). Econometría. México: McGraw-Hill.

Hodgson, G., \& Jiang, S. (2008, primer semestre). La economía de la corrupción y la corrupción de la economía: una perspectiva institucionalista. Revista de Economía Institucional, 10(18), 55-80.

Huntington, S. P. (1968). Political order in changing societies. New Haven: Yale University Press.
Instituto Mexicano para la Competitividad-IMCO. (2015). Índice de Competitividad Internacional 2015. La corrupción en México: Transamos y no avanzamos. México: IMCO.

Jain, A. K. (2001). Corruption: A review. Journal of Economics Surveys, 15(1). 71-120.

Klitgaard, R., MacLean-Abaroa, R., \& Parris, H. (2000). Corrupt Cities: A Practical Guide to Cure and Prevention. Oakland, California: ICS Press and World Bank Institute.

Leff, N. (1964). Economic development through bureaucratic corruption. En American Behavioral Scientist, pp. 8-14.

Martín-Baumeister, B. (2014). La corrupción en el sector privado. Revista Crítica, 29-32.

Morillo, A. (2009). La Corrupción, ¿problema social o político? Revista Venezolana de Ciencia Política, 36, 147-160.

North, D. (1993). Instituciones, cambio institucional y desempeño económico. Chile: FCE.

Sasia, P. (2014). Entender y combatir la corrupción en estos tiempos. Revista Crítica, 20-24.

Transparencia Internacional. (2013). Corruption perception index. Recupeado de http://cpi.transparency.org/cpi2013/in_detail/\#myAnchor1

Zakiuddin, A. (2001). Corruption in Bangladesh. An analytical and sociological study. Documento preparado para Transparencia Internacional, Bangladesh, Bangkok. Recuperado de http://unpan1.un.org/intradoc/groups/public/documents/ apcity/unpan004881.pdf 